ist dieser Befund ein ethisch relevantes Ergebnis ${ }^{52}$. Wirtschaftskriege sind also nicht nur rechtlich verboten, sie sind auch aus ethischer Perspektive abzulehnen.

52 Zumindest hinzuweisen ist auf die Gefahr, dass eine solche Argumentation die Privatisierung und Finanzialisierung der Gewalt unterstützen könnte (vgl. dazu Erhard Eppler, Vom Gewaltmonopol zum Gewaltmarkt? Die Privatisierung und Kommerzialisierung der Gewalt, Frankfurt/Main 2002). Private Gewaltunternehmer könnten sich als Alternative zum staatlichen Gewaltmonopol profilieren und damit könnte Sicherheit von der Finanzkraft privater Akteure abhängig werden. Mit dieser Entwicklung würde das wirtschaftliche Paradigma über die Politik dominieren. Daher bleibt es Aufgabe der Politik, ihr Gewaltmonopol nicht aushöhlen zu lassen - bspw. durch klare Rahmenbedingungen. Und an wirtschaftliche Unternehmen müsste die Frage gestellt werden, ob sie sich nicht dieser Entwicklung selbst entgegenstellen sollten, denn eine Schwächung des staatlichen Gewaltmonopols würde in der Folge auch die Unternehmen treffen.

\title{
Europäische Migrations- und Außengrenzpolitik - Wege aus der Sackgasse?
}

\section{Ruth Vollmer*}

\begin{abstract}
Increasingly challenged by a growing number of deaths in the Mediterranean and critical public debates, EU policy makers continue their more-of-the-same approach regarding external border policies, turning to the prevailing security-focussed paradigm for a solution. This paper argues that such an approach will do nothing to solve the problem of deadly and inhumane external borders, but may well exacerbate it. Although conceptions of migration vary (development potential, demographic necessity, security risk), the European migration and border regime appears to have developed a self amplifying dynamic, while real alternatives seem politically blocked. This article argues that central assumptions underlying the current approach do not hold and suggests policy alternatives, which would strengthen coherence between the EU's border policies and human rights agenda taking into account inherent tensions between migration and the (welfare) state.
\end{abstract}

Keywords: EU, borders, migration, externalizaton;

EU, Grenzen, Migration, Externalisierung

\section{Einleitung}

Am 3. Oktober 2013 starben weniger als eine Meile vor der Küste Lampedusas mehr als 360 Menschen aus Eritrea und Somalia, nachdem ihr Boot mit insgesamt ca. 500 Menschen an Bord nach 13 Tagen auf See in Brand geraten war und sank. Eines von drei Fischerbooten, die sich während des Brandes in Sichtweite des Schiffes aufhielten, leistete Hilfe und konnte 47 der Flüchtlinge retten, jedoch erst nachdem deren Boot bereits gekippt war und alarmierte auch erst dann die Küstenwache, die 45 Minuten später eintraf. Die Geretteten wurden zu einer Geldstrafe wegen „illegaler Einwanderung“ verurteilt und zunächst im Auffanglager in Lampedusa, das damals etwa viermal so viele Personen beherbergte, wie vorgesehen, interniert; die Verstorbenen erhielten ein Staatsbegräbnis. ${ }^{1}$

* $\quad$ Ruth Vollmer hat 2008 ihr Magisterstudium der Politikwissenschaft, Sprachwissenschaft und Psychologie an der Universität Bonn abgeschlossen; sie arbeitet derzeit am Internationalen Konversionszentrum Bonn (BICC). Dieser Artikel wurde einem anonymisierten Begutachtungsverfahren unterzogen (double-blind peer-reviewed). Die Autorin bedankt sich bei den Gutachtern für wertvolle Hinweise und hilfreiche Kommentare.

1 Perkowski, Nina (2013): No accident. http://opendemocracy.net/5050/ nina-perkowski/no-accident-why-have-19142-died-at-europe\%E2\%80\%99sfrontiers (eingesehen am 17.1.14)
Lampedusa, eine kleine italienische Mittelmeerinsel mit ca. 5.000 Einwohnern, die geografisch näher an Tunesien als an Italien liegt, ist seither umso mehr zu einem Synonym für das Sterben von Menschen an Europas Außengrenzen geworden. Denn anders als unzählige vorhergehende ähnliche Vorfälle ${ }^{2}$ sorgte diese „Tragödie“, möglicherweise aufgrund ihrer Nähe zu Europa und der vielen Toten, für ein außergewöhnlich starkes Medienecho und einen „unprecedented call for action by EU leaders and citizens, “3 der darauf zielte, Ähnliches in Zukunft zu verhindern.

Zwei Monate nach „Lampedusa“ legte die Task Force for the Mediterranean ihren Bericht vor, den die zuständige EUKommissarin als eine "truly European response that can make a difference ${ }^{\prime 4}$ ankündigte. Darin wird ein integrierter Ansatz

2 Die Internationale Organisation für Migration schätzt, dass seit 199320.000 Menschen an den europäischen Außengrenzen ums Leben gekommen sind. Solche Zahlen stehen für eine vermutlich deutlich höhere Dunkelziffer. http:// www.iom.int/cms/render/live/en/sites/iom/home/news-and-views/pressbriefing-notes/pbn-2013/pbn-listing/iom-mourns-lampedusa-shipwreck-v. html (eingesehen am 17.1.14).

3 European Commission (2013): Communication from the European Commission the European Parliament and the Council on the work of the Task Force Mediterranean. COM(2013) 869 final. Brüssel.

4 Malmström, Cecilia (2013): Preventing deaths in the Mediterranean. http://ec.europa.eu/commission_2010-2014/malmstrom/news/ archives/2013/12/20131204_en.htm (eingesehen am 17.1.14). 
für den gesamten Mittelmeerraum gefordert. Gemäß dem „Global Approach towards Migration and Mobility“ soll die Zusammenarbeit mit Herkunfts- und Transitstaaten ausgeweitet werden, wobei ein Schwerpunkt auf die Umsetzung bestehender und Aushandlung neuer „Mobilitätspartnerschaften“ gelegt wird. In Dialogprozessen soll zudem eine Verbesserung der Rechtslage und Verwaltungspraxis zu Asyl und Migration sowie zur Menschenrechtslage in Transitstaaten erzielt und gleichzeitig Kapazitäten zur Grenzkontrolle und Bekämpfung von Menschenschmuggel und Menschenhandel gestärkt werden. Weitere Vorschläge umfassen Kampagnen zur Aufklärung über die Gefahren irregulärer Migration, verbesserten Informationsaustausch mit den südlichen Mittelmeerstaaten, mehr Patrouillen in Drittstaatengewässern, den Ausbau regionaler Schutzprogramme für Flüchtlinge, die Überprüfung legaler Einreisewege in die EU, eine verstärkte Beteiligung von EUStaaten an dem Neuansiedlungsprogramm des UNHCR, dem Kampf gegen Menschenschmuggel und Menschenhandel, schnelle, dauerhafte und menschenwürdige Rückführungen, die Stärkung des Außengrenzmanagements, umfassende und koordinierte Grenzüberwachungsoperationen im Mittelmeer unter der Anleitung von Frontex, die Weiterentwicklung und stärkere Nutzung der EUROSUR-Kapazitäten zur Seenotrettung, (finanzielle) Hilfe und Solidarität mit den südlichen Mitgliedstaaten und verstärkte europäische Zusammenarbeit. Die Einhaltung geltender menschen- und flüchtlingsrechtlicher Verträge bei allen entsprechenden Maßnahmen wird angemahnt. ${ }^{5}$

Im Folgenden wird dieser Beitrag die derzeitige europäische Außengrenzpolitik beschreiben, um diese Reaktion auf „Lampedusa“ in einen breiteren Kontext einzuordnen. Anhand der darzustellenden Auswirkungen dieser Politik kann dann ihr Begründungszusammenhang problematisiert und anschließend gezeigt werden, worin selbstverstärkende Momente dieses Regimes bestehen. Der letzte Teil geht auf mögliche und derzeit diskutierte Alternativen ein.

\section{Das europäische Migrationskontrollregime}

Von allen Bereichen europäischer Migrationspolitik, die laut Europäischem Pakt zu Einwanderung und Asyl so unterschiedliche Ziele wie die Gestaltung legaler und die Bekämpfung irregulärer Einwanderung, die Stärkung der Außengrenzen, die Umsetzung des gemeinsamen europäischen Asylsystems und die Entwicklung einer umfassenden Partnerschaft mit Herkunfts- und Transitländern für Migration und Entwicklung umfasst, gestaltete sich die sogenannte externe Dimension der Migrationskontrolle und Stärkung der Außengrenzen besonders dynamisch. Handlungsleitend ist hier seit den frühen 1990er Jahren die Annahme, unkontrollierte Migration könne eine Gefahr für die Zielländer darstellen und sei zu verhindern. ${ }^{6}$ Die durch die Aufhebung der Binnengrenzkontrollen im Rahmen der Schengener Abkommen erfolgte Aufwertung der europäischen Außengrenzkontrollen (das Durchführungsabkommen trat 1995 in Kraft) zog einen deutlichen Anstieg

5 Zu alledem: European Commission, 2013, a.a.O.

6 Lavenex, Rahel und Kunz, Sandra (2008): „The Migration-DevelopmentNexus in EU External Relations." European Integration, Vol. 30, No 3, S. 439-457. entsprechender Maßnahmen nach sich. Durch den Vertrag von Amsterdam (1999), der den „Raum der Freiheit, der Sicherheit und des Rechts“ begründete, wurden migrationspolitische Entscheidungen der EU überwiegend in der 1. Säule vergemeinschaftet; seither können EU-Institutionen diesbezüglich selbst mit Drittstaaten verhandeln. Ausgenommen blieb davon der Bereich legaler (Arbeits-)Migration, der vollständig in der Kompetenz der Mitgliedstaaten verblieb (einstimmiger statt Mehrheitsbeschluss im Ministerrat). ${ }^{7}$ Die besondere Dynamik der externen Dimension auf europäischer Ebene liegt somit zum einen in der gesteigerten Bedeutung der Außengrenzkontrollen und zum anderen im Fehlen eines Mandats zur Ermöglichung legaler Migration.

In der Praxis weisen europäische Außengrenzkontrollen eine Vervielfachung von Akteuren auf. Private Akteure übernehmen vormals staatliche Aufgaben, wie beispielsweise Anbieter von Sicherheitsdienstleistungen, die Passagierkontrollen durchführen, sog. Aufnahmeeinrichtungen leiten oder Abschiebungen unterstützen. Hersteller von Sicherheitstechnologien entwickeln Produkte und Lösungen für die zunehmend technologisierte Überwachung und Kontrolle von Grenzen. Beförderungsunternehmen sind ohnehin seit Schengen veranlasst, ausschließlich Personen mit gültigem Visum zu transportieren. Internationale staatliche und nichtstaatliche Organisationen spielen eine immer größere Rolle, wie beispielsweise die Internationale Organisation für Migration (IOM) ${ }^{8}$ die u.a. Rückkehrprogramme unterstützt. Verschwimmende Grenzen zwischen innerer und äußerer Sicherheit, zivilen und militärischen Akteuren, lassen sich ebenfalls anhand der wachsenden Akteursvielfalt mit Blick auf Grenzkontrollen nachzeichnen. Die im Rahmen der Europäisierung erforderlich gewordene Koordination der Zusammenarbeit führte letztlich auch noch zu der Befassung einer Fachagentur mit der Thematik (Frontex).

Daneben kennzeichnet die Praxis der Außengrenzkontrollen eine Vervielfältigung von Orten und Technologien. ${ }^{9}$ Europäische Außengrenzkontrollen haben sich vom geografischen Grenzverlauf gelöst und an verschiedenste Orte, in Herkunftsund Transitstaaten, ins Inland, internationale und Drittstaatsgewässer, im Rahmen der Technologisierung auch bis in den Weltraum verlagert. Die Einbindung süd- und osteuropäischer Staaten in die Migrationskontrolle begann unmittelbar nach dem Fall des „Eisernen Vorhangs“. ${ }^{10}$ Im Rahmen der Verhandlungen zum Cotonou-Abkommen wurden diese Bemühungen auf Nordafrika ausgeweitet ${ }^{11}$ und mit der Verabschiedung des „Global Approach to Migration“ (seit 2011 Global Approach to Migration and Mobility - GAMM) wurde der Fokus auf ex-

7 Parkes, Roderick und Angenendt, Steffen (2009): The re-nationalisation of migration policy-making? EU cooperation after the Immigration Pact. Working Paper FG 1, 2009 / 02 and FG 8, 2009 / 05, SWP: Berlin.

8 Geiger, Martin und Pécoud, Antoine (2012): "The New Politics of International Mobility. Migration Management and its Discontents." IMIS-Beiträge, No. 40, S. $11-22$.

9 Tholen, Berry (2010): "The changing border: developments and risks in border control management of Western countries." International Review of Administrative Science, Vol. 76, No. 2, S. 259-78.

10 Schwenken, Helen (2007): „Die Herstellung von Illegalität - Das Scheitern von Migrationskontrolle ist kein Zufall.” In Heinrich Böll Stiftung (Hg.). Leben in der Illegalität. Ein Dossier. Schriften zur Demokratie, Band 5, S. 16-22.

11 Van Criekinge, Tine (2010): „The EU-Africa migration partnership: a case study of the EU's migration dialogue with Ghana and Senegal." Paper prepared for the EUI Migration Working Group. European University Institute, Florence, Italy, March. 
terne Aktivitäten weiter vertieft, mit den Staaten des südlichen Mittelmeers als Priorität. ${ }^{12}$ Die Multiplizierung der Technologien bezeichnet den zunehmenden Einsatz von Sicherheitstechnologien wie unbemannte Drohnen, Überwachungs- und Sensortechnologien und biometrische Erkennungsprogramme in der Beobachtung von Migrationsbewegungen und der Ausrüstungen von Grenzanlagen. Sie werden ergänzt durch umfangreiche Vorhaben zur Datenspeicherung, z. B. EURODAC (European Dactyloscopy), eine Datenbank personenbezogener Daten von Asylbewerber/-innen und undokumentierten Einwanderern. ${ }^{13}$ EUROSUR (European Border Surveillance System), das zivil-militärische Grenzüberwachungssystem der EU, steht exemplarisch für alle drei Entwicklungen. Es ging im Dezember 2013 in sieben Mitgliedstaaten der EU in Betrieb und sorgt für die Vernetzung aller Grenzbehörden und migrationsbezogenen Datenbanken der Mitgliedstaaten sowie von Frontex und erleichtert den Informationsaustausch zwischen unterschiedlichsten Überwachungstechnologien von Satelliten über Radar bis hin zu Drohnen. ${ }^{14}$ Die Smart Borders Initiative der EU-Kommission verfolgt das Ziel, die Grenzüberquerungen sogenannter Bona-fide-Reisender (nach einem freiwilligen Prüfverfahren registrierte Drittstaatsangehörige $)^{15} \mathrm{zu}$ vereinfachen und mit dem elektronischen Ein- und Ausreisesystem (EES) gleichzeitig Visumsüberschreitungen zu erkennen und verfolgen.

Wie insbesondere das letzte Beispiel zeigt, dient die Praxis der Außengrenzkontrollen nicht der vollständigen Verhinderung von Einwanderung, sondern vielmehr der Durchsetzung einer im Vorfeld auf politischer Ebene vorgenommenen Selektion zwischen erwünschter (z.B. hochqualifizierte Personen) und unerwünschter Einwanderung. ${ }^{16} \mathrm{Zu}$ letzterem gehören Familiennachzug und die Beantragung von Asyl oder humanitärem Schutz als juristische Kategorien, in deren Rahmen eine Einreise rechtlich zumindest vorgesehen ist, sowie jede andere (rechtlich nicht vorgesehene Form) von Arbeitsmigration, u.a. . ${ }^{17}$ Entscheidend für diese Differenzierung ist,

12 Hayes, Ben. (2009): Neoconopticon. The EU Security-Industrial Complex. London: Statewatch and Transnational Institute. http://www.statewatch.org/analyses/ neoconopticon-report.pdf (eingesehen am 17.1.14).

13 Scheffran, Jürgen und Vollmer, Ruth (2012): Migration und Klimawandel: globale Verantwortung der EU statt Angstdebatte. In Bruno Schoch, Corinna Hauswedell, Janet Kursawe, Margret Johannsen (Hrsg.): Friedensgutachten 2012. Berlin: LIT Verlag, S. 209-221.

14 Monroy, Matthias (2011): Militarisierung des Mittelmeers. http://www.heise. de/tp/artikel/34/34515/1.html (eingesehen am 17.1.14).

15 Europäische Kommission (2011): Mitteilung zur Migration, Brüssel. http:// ec.europa.eu/commission_2010-2014/president/news/speeches-statements/ pdf/20110504_de.pdf (eingesehen am 17.1.14).

16 Vogelskamp, Dirk (2008): „Die Unerwünschten. Verwaltung der Migration: Überwachung, Kontrolle und repressive Exklusion." Wissenschaft und Frieden, Vol. 26, No. 2, S. 11-14. Vgl. auch Purtschert, Patricia und Meyer, Katrin (2008): „Migrationsmanagement und die Sicherheit der Bevölkerung.“ In: Patricia Purtschert, Katrin Meyer und Yves Winter (Hrsg.): Gouvernementalität und Sicherheit. Zeitdiagnostische Beiträge im Anschluss an Foucault. Bielefeld: transcript, S. 149-172. Die Autorinnen betonen, dass die Unterscheidung zwischen regulärer und irregulärer Migration rechtlichen Normen folgt, „welche der Prüfung von Asylgesuchen vorgelagert sind." Rechtlich an sich vorgesehene Migrationswege werden also u.a. durch die Externalisierung der Migrationskontrollen geschlossen, bevor die Betroffenen ihren Rechtsanspruch geltend machen können. Im Fall von Familiennachzug ist die Einführung des Sprachtests 2007 eine Maßnahme, die de facto diese Wirkung erzielt, vgl. dazu: Biermann, Sybille (2014): Familiennachzug. „Ich dachte, wir leben in einem Rechtsstaat. " http://www.migazin.de/2014/02/04/ familiennachzug-visum-eugh-deutschland-rechtsstaat/ (eingesehen am 6.2.2014).

17 Buckel, Sonja und Wissel, Jens (2010): "State Project Europe: The Transformation of the European Border Regime." International Political Sociology, Vol. 4, No. 1, S. 33-49. dass sich „unerwünschte“ Formen der Einwanderung in der Vergangenheit als von staatlicher Seite weder steuerbar noch kalkulierbar erwiesen haben und auf ihre Unkalkulierbarkeit im Politikmodus der „Gefahrenabwehr“ reagiert wird, der sich auch über die europäische Ebene manifestiert. ${ }^{18}$ Es hat sich ein Regime entwickelt, das aufgrund der Akteursvielfalt in teilweise sehr intransparenter Weise mithilfe eines hochmodernen Sicherheitsinstrumentariums unkontrollierte Migration beinahe weltweit zu unterbinden sucht. Die Gesamtheit finanzieller Mittel, welche die EU für diese Art der Grenzsicherung und die Rückführung unerwünschter Personen aufwendet, ist indes schwer zu beziffern, da die Kosten in sehr unterschiedlichen Bereichen anfallen. Zwischen 2007 und 2013 flossen nach Kommissionsangaben mehr als 1,8 Milliarden Euro in den europäischen Außengrenzenfonds, wohingegen für den Fonds zur Integration von Drittstaatsangehörigen lediglich 825 Millionen Euro bereitgestellt wurden. ${ }^{19}$

\section{Die Lösung als Problem?}

In diesem Kontext fand also die Arbeit der Task Force for the Mediterranean statt. Ihr Bericht greift in der Mehrzahl der Punkte auf bestehende oder ohnehin geplante Instrumente zurück. Dies gilt für die Verstärkung von Kontroll- und Überwachungsinstrumenten, die nun auch der Seenotrettung dienen sollen, für die vertiefte Kooperation mit Drittstaaten, die Maßnahmen zur Bekämpfung von Menschenschmuggel und Menschenhandel und ebenso für regionale Schutzprogramme und den Ruf nach mehr Neuansiedlungen von Flüchtlingen. Die zur Verhinderung von Todesfällen an den Außengrenzen erarbeiteten Vorschläge laufen auf eine Intensivierung und Ausweitung des bestehenden Regimes hinaus, was angesichts der Zusammensetzung der Task Force wenig erstaunt: Unter der Leitung der Kommission tagte der Europäische Auswärtige Dienst mit Vertretern und Vertreterinnen von Mitgliedstaaten und Agenturen wie Frontex, Europol und EASO. Was die Maßnahmen betrifft, die für eine Abkehr vom bisherigen Kurs zu stehen scheinen, hier vor allem die Überprüfung sicherer, legaler Einreisewege, stehen die institutionellen Voraussetzungen für eine Reform ungünstig. Vor allem Deutschland hat sich in der Vergangenheit bei dieser Frage gegen qualitative Mehrheitsentscheide im Ministerrat ausgesprochen; Vorstöße der Kommission zur legalen Einwanderung wurden von den Mitgliedstaaten abgeblockt. ${ }^{20}$ Während die Vergabe humanitärer Visa für Asylsuchende rechtlich bereits möglich ist, aber nicht praktiziert wird, beweist der Verweis auf die Direktiven zu Saisonarbeit und Bildungsmigration (noch in Verhandlung) keine über die bestehenden Möglichkeiten hinausgehenden Ambitionen der Verfasser.

Eine solche Reaktion ist nicht geeignet, den angekündigten „Unterschied zu machen“. ${ }^{21}$ Das zeigt auch ein Blick zurück. Der GAMM selbst, der den Rahmen für die geplanten Schritte darstellt, wurde 2005 unter dem Eindruck der Vorfälle in

18 Bommes, Michael (2011): „Die Planung der Migration.“ IMIS-Beiträge 38/2011, S. $115-136$

19 Angaben laut Homepage der Generaldirektion Inneres: http://ec.europa eu/dgs/home-affairs/financing/fundings/migration-asylum-borders/index en.htm (eingesehen am 26.1.2014)

20 Lavenex und Kunz, 2008, a.a.O.

21 Malmström, Cecilia, 2013, a.a.O. 
Ceuta und Melilla verabschiedet, wo mehrere Flüchtlinge bei dem Versuch, die Grenzzäune um die spanischen Exklaven in Marokko zu überwinden, von Grenzschützern erschossen worden waren. Um Ähnliches in Zukunft zu verhindern wurde damals u. a. die Ausweitung von Überwachungs- und Kontrollmaßnahmen beschlossen sowie besagter "globaler Ansatz“, der Herkunfts- und Transitstaaten mit einbeziehen soll. Dass dies nicht dazu beitragen würde, die Sicherheit von Flüchtlingen zu erhöhen, wurde schon damals vorhergesagt. ${ }^{22}$

Vielmehr konnte bereits gezeigt werden, dass die Ursachen der unsicheren und tödlichen Migrationsrouten in den Systemen liegen, die nun auf ein Neues zu ihrer vermeintlichen Lösung vorangetrieben werden. Seit 1991 wird die Reise- und Zirkulationsfreiheit von Menschen zwischen Nordafrika und Europa einseitig durch die Einführung der Visumspflicht illegalisiert, ${ }^{23}$ Maßnahmen zur Verhinderung der nun irregulär gewordenen Migration werden seither wie gezeigt stetig ausgebaut. Die dadurch erschwerten und unsicheren Reisebedingungen führen in der Folge zu höheren Risiken für Migrantinnen und Migranten und stärkerer Abhängigkeit von Schleusern, ${ }^{24}$ dies wiederum zu mehr Toten - und diese zur weiteren Verschärfung der Kontrollen, einem Ausbau der Grenzüberwachungssysteme und Verstärkung der Strafverfolgung. ${ }^{25}$ Selbst wenn man die Toten als Teil einer kalkulierten Abschreckungsstrategie der EU betrachtet, ${ }^{26}$ wird klar, dass diese nicht aufgeht. Die irreguläre bzw. illegalisierte Migration findet nicht nur trotz abschreckender Bilder, hoher Risiken und teurer Gegenmaßnahmen weiterhin statt, sondern teilweise gerade deswegen. Für die in nahezu allen Einwanderungsstaaten feststellbare „signifikante Lücke zwischen programmatischen Zielen und tatsächlichen Folgen der Migrationspolitiken "27 bestehen unterschiedliche Erklärungsmodelle. Als bedeutsame Faktoren gelten etwa „divergierende innenpolitische Interessen“, die sich in politischen Inkohärenzen niederschlagen, die anhaltende Nachfrage nach Arbeitskräften, gegenüber denen geringere rechtliche Standards gelten, die sogenannte Kettenmigration, die sich über familiäre und soziale Netzwerke staatlichen Interventionen zum Trotz fortsetzt, sowie rechtstaatliche Grundsätze und internationale Menschenrechtsregime, welche die Handlungsfähigkeit der

22 Spijkerboer, Thomas (2007): “The Human Costs of Border Control." European Journal of Migration and Law, 9 (2007), S. 127-139.

23 Einreisen zum Zweck des dauerhaften Aufenthalts waren zuvor zwar ebenso wenig vorgesehen, grundsätzlich waren Einreisen jedoch nicht nur im Rahmen von Anwerbeprogrammen, sondern auch generell leichter möglich und viele Menschen konnten einen dauerhaften Aufenthaltsstatus nachträglich realisieren, u.a. durch die Legalisierungskampagnen in mehreren europäischen Staaten in den 1970er und 80er Jahren, vgl. de Haas, Hein (2007): North African Migration Systems: evolution, transformations and development linkages. IMI Working Papers, No. 6.

24 Es ist in diesem Zusammenhang wichtig, zwischen Schleusern, die Menschen überwiegend auf ihren eigenen Wunsch hin und gegen Bezahlung befördern, und Menschenhändlern zu unterscheiden. Letztere verwenden Gewalt und andere Druckmittel, um Menschen auszubeuten. Der Task-Force-Bericht macht diese Unterscheidung zwar, verwendet die Begriffe aber ansonsten fast durchgängig in Verbindung zueinander, was nicht vorhandene Ähnlichkeit suggeriert.

25 de Haas, Hein (2013): Lampedusa. Only the dead can stay. http://heindehaas. blogspot.de/2013/10/lampedusa-only-dead-can-stay.html (eingesehen am 17.1.14).

26 Heinbach, Gesa (2013): „Lampedusa: Europas Schande.“ Blätter für deutsche und internationale Politik, 11/2013, S. 5-8.

27 Benz, Martina und Schwenken, Helen (2005): „Jenseits von Autonomie und Kontrolle. Migration als eigensinnige Praxis." PROKLA 140, S. 363-377.
Staaten bezüglich Gegenmaßnahmen limitieren. ${ }^{28}$ Vereinfacht lässt sich jedoch festhalten, dass solange legale Einreisewege versperrt sind, solche Maßnahmen das Phänomen, zu dessen Bekämpfung sie eingeführt wurden, verstärken.

Es steht also zu erwarten, dass durch den Ausbau und die Vertiefung des derzeitigen Migrationsregimes in erster Linie auch die damit verbundenen problematischen Folgen verstärkt werden, während eine Lösung in weitere Ferne rückt. Dass die bessere Überwachung des Mittelmeers nicht automatisch zur Rettung von mehr Menschenleben führen wird, zeigt eindrücklich die Odyssee eines Flüchtlingsboots, das im März 2011 während des NATO-Einsatzes in Libyen in einem der damals mit am besten überwachten Meeresgebiete überhaupt 15 Tage lang manövrierunfähig vor sich hin trieb. 63 der 72 Insassen starben, obwohl die Notrufe mit den Koordinaten des Bootes immer wieder an alle Schiffe der Umgebung gingen. ${ }^{29}$

Gefahr für Leib und Leben droht Migrantinnen und Migranten unterdessen nicht nur durch Schleuserabhängigkeit und immer gefährlichere Routen. 2013 deckten Nichtregierungsorganisationen teilweise brutale Menschenrechtsverletzungen durch Grenzschützer ${ }^{30}$ auch im Rahmen von Frontex-Operationen auf. Obwohl seit 2011 eine Menschenrechtsbeauftragte die Operationen von Frontex überwacht, räumte der Leiter der Agentur 2013 Reportern gegenüber erstmals auch die Beteiligung an sog. Push-back-Operationen ein, bei denen Menschen ohne Möglichkeit einen Asylantrag zu stellen abgewiesen oder zurückgeschoben werden (was bis dahin stets in den alleinigen Verantwortungsbereich der Mitgliedstaaten verwiesen wurde). ${ }^{31}$

Die Einbindung von Drittstaaten, die von EU-Seite im Rahmen diverser Kooperationsprogramme, der Europäischen Nachbarschaftspolitik, Beitrittsverhandlungen, bi- und multilateralen (Handels-)Abkommen sowie Wirtschaftshilfen vorangetrieben wird, zielt vor Ort vor allem auf die Verstärkung der Kontrollen der in einigen Staaten mittlerweile illegalisierten Ausreisen und die regionale Versorgung von Flüchtlingen ab. Gerade mit Blick auf Nordafrika hat die Auslagerung der Migrationskontrolle die Situation von Flüchtlingen und Migrantinnen aus Subsahara-Afrika zum Teil dermaßen verschlechtert, dass eine Weiterreise Richtung Europa zunehmend überlebensnotwendig wird. ${ }^{32}$ Die bedenkliche Menschenrechtslage in Transitstaaten, die nun durch Dialog mit diesen Staaten verbessert werden soll, ist nicht zuletzt eine Konsequenz der externalisierten Migrationskontrollen. Die Mobilitätspartnerschaften, von einem NGO-Bündnis jüngst als Immobilitätspartnerschaften

28 Ebenda. Die Autorinnen kritisieren an diesen Ansätzen deren Staatszentriertheit und das Ausklammern anderer wichtiger Faktoren; auch für den vorliegenden Beitrag gilt, dass er diese Perspektiven zu wenig abbildet.

29 Council of Europe Parliamentary Assembly (2012): Lives lost in the Mediterranean Sea. Who is responsible? http://assembly.coe.int/ CommitteeDocs/2012/20120329_mig_RPT.EN.pdf (eingesehen am 17.1.14)

30 Pro Asyl (2013): pushed back. systematic human rights violations against refugees in the aegean sea and at the greek-turkish land border. http://www.proasyl. de/fileadmin/fm-dam/1_EU_Fluechtlingspolitik/pushed_back_web_01.pdf (eingesehen am 17.1.14).

31 Der Videobeitrag und das Manuskript der Sendung sind unter folgendem Link abrufbar: http://www.wdr.de/tv/monitor/sendungen/2013/1017/europa. php5 (eingesehen am 3.2.2014); zur menschenrechtlichen Bewertung sog. Push-Back-Operationen EGMR, Urteil der Großen Kammer vom 23. Februar 2012, Application no. 27765/09, Hirsi Jamaa und andere v. Italien.

32 de Haas, 2013, a.a.O. 
bezeichnet, ${ }^{33}$ haben in der Tat ihr Versprechen einen Beitrag zur Mobilität von Menschen aus den „Partnerländern“ zu leisten, bisher nicht eingehalten. Sie fordern zunächst den Aufbau stärkerer Migrationskontrollkapazitäten, um dann - die bilateral auszuhandelnde Bereitschaft einzelner EU-Mitgliedstaaten vorausgesetzt - Migration zuzulassen. ${ }^{34}$

Eine der aus menschenrechtlicher Sicht bedenklichsten Folgen der Externalisierung ist die weitere Aushöhlung des Rechts auf Asyl. Politisch Verfolgte werden kriminalisiert und ihnen wird es zunehmend unmöglich gemacht, dahin zu gelangen, wo sie Asyl beantragen könnten. Auch das jüngst in Kraft getretene Rücknahmeabkommen mit der Türkei weist in diese Richtung, da es EU-Staaten erlaubt, über die Türkei eingereiste Flüchtlinge ohne Prüfung ihrer Schutzbedürftigkeit wieder zurückzuschieben, obwohl nach türkischem Recht nur Bürgerinnen und Bürger europäischer Staaten dort asylantragsberechtigt sind, syrische Flüchtlinge bspw. können bestenfalls ein zeitlich begrenztes Bleiberecht erhalten. ${ }^{35}$

\section{Eigendynamiken und Widersprüche}

Das aktuell praktizierte Migrationsregime der EU verstößt gegen in der EU geltende Menschenrechtsstandards, insofern z. B. eine Prüfung der individuellen Gefahr der Folter oder unmenschlichen Behandlung im Zielstaat nicht ermöglicht wird, ${ }^{36}$ und es verschlimmert die Probleme, die es laut seinen Befürwortern beheben soll. Wie kommt es trotz der ins Auge springenden Unzulänglichkeit des aktuellen Regimes zu solch selbstverstärkenden Momenten, wie sie immer wieder - gerade in kritischen Phasen nach dem sog. „Arabischen Frühling“ oder "Lampedusa“ - zu beobachten sind? Eine Reihe von Faktoren spielt dabei eine Rolle.

In der europäischen Migrations- und Außengrenzpolitik manifestieren sich die Interessen und Wahrnehmungen diverser Akteure, sie ist immer als Ergebnis komplexer Aushandlungsprozesse zu sehen. Infolgedessen existieren unterschiedliche und teilweise widersprüchlich Bewertungen und Konzeptionalisierungen von Migration. Sie kann als überlebensnotwendig für die europäischen Renten- und Sozialsysteme, als wichtiger Beitrag gegen Fachkräftemangel, als Entwicklungsmotor für die Herkunftsländer oder als tendenziell bedrohliche Begleiterscheinung von Globalisierung betrachtet werden, verbunden jeweils mit einem spezifischen Set als relevant erachteter Fakten und einer normativen Bewertung. Welche(s) framing(s) sich letztendlich maßgeblich auf die Ausarbeitung und Umsetzung konkreter Politiken auswirkt, entscheidet nicht zuletzt das Machtgefüge zwischen den beteiligten Akteuren. ${ }^{37}$ Insofern gilt auch für Reformansätze dieser Politik, dass diese nicht in

33 Brot für die Welt, medico international, Pro Asyl (Hrsg.) (2013): Im Schatten der Zitadelle. Der Einfluss des Europäischen Migrationsregimes auf "Drittstaaten." http://www.proasyl.de/fileadmin/fm-dam/NEWS/2013/Im_Schatten_der Zitadelle_2013.pdf (eingesehen am 3.2.2014).

34 Ebenda.

35 Vgl. http://www.reuters.com/article/2013/04/12/us-turkey-refugeesidUSBRE93B0XO20130412 (eingesehen am 3.2.2014).

36 EGMR, Urteil der Großen Kammer vom 23. Februar 2012, Application no. 27765/09, Hirsi Jamaa und andere v. Italien.

37 Lavenex und Kunz, 2008, a.a.O. einem Vakuum entstehen, sondern immer auf bereits etablierte Ansätze und institutionelle Rahmenbedingungen treffen.

Den Merkmalen des bisher einflussreichsten framings von Migration ist Krause ${ }^{38}$ nachgegangen, auch um zu zeigen, wie es die Art des Sprechens über Migration ermöglicht, über das Sterben an den Außengrenzen, von einzelnen Vorfällen abgesehen, mit bemerkenswerter Gleichgültigkeit hinwegzugehen. Dabei handelt es sich zunächst um die Konstruktion von Migration als „Anomalie und Problem", ungeachtet der Tatsache, dass Migration für die Menschheit seit jeher vielmehr den Normalzustand als die Ausnahme darstellt. Im nächsten Schritt folgt die Kriminalisierung und Illegalisierung der Migration. Migrantinnen und Flüchtlinge werden als Problem begriffen und enger Regulierung und Kontrolle unterworfen. Nicht nur über die Naturkatastrophen- und Wassermetaphorik (Flucht und Migration als Strom, Lawine, Tsunami, etc.) wird der Topos von „Migration als Invasion“ etabliert. Er steht exemplarisch für eine Auffassung, derzufolge Migration nicht nur unkontrollierbar und massenhaft erfolgt, sondern vor allem arme Bevölkerungsschichten betrifft ${ }^{39}$ und unterstreicht zudem das von ihr ausgehende Bedrohungspotenzial für Europa. " ${ }^{40}$ Diesen Mythos einer Invasion zu reproduzieren, der im letzten Schritt in einer Verteidigungsrhetorik gipfelt, mag aus Sicht seiner Vertreter kurzfristig Vorteile versprechen - vom Ablenken von innenpolitischen Problemen, bis hin zu einer dauerhaften Daseinsberechtigung für Akteure wie Frontex. Gleichzeitig verbaut sich die entsprechende Politik so die Möglichkeit einer notwendigen und nachhaltigen Reform der Einwanderungspolitik, indem sie die notwendige öffentliche Unterstützung untergräbt. ${ }^{41}$

Weltweit werden derzeit Wohlstandsunterschiede durch Grenzen und Zäune zementiert. Dieses „re-bordering“ steht in Kontrast zu dem seit Jahrzehnten anhaltenden Trend der abnehmenden Bedeutung von Grenzen (de-bordering) für Warenverkehr, Wirtschafts- und Finanzbeziehungen. In einigen Fällen, wie dem von der Airbus Group (ehem. EADS) um Saudi-Arabien herum errichteten hochtechnisierten Grenzzaun, bilden Sicherheitsrisiken einen wichtigen Anlass für den Bau. In anderen hingegen helfen vermeintliche Sicherheitsrisiken diese Abschottung nachträglich zu begründen, sind jedoch nicht von vornherein gegeben. Dies trifft auf die EU mit ihren Zäunen an der griechisch-türkischen Landgrenze, um ihre Exklaven Ceuta und Melilla und zunehmend deterritorialisierte Grenzkontrollen ebenso zu wie auf die USA mit ihrem Grenzzaun zu Mexiko und zunehmenden Überwachungsaktivitäten an der Grenze zu Kanada,. ${ }^{42}$ Das re-bordering, die Schließung und Absicherung von Grenzen zieht ihre eigene Normalisierung und Legitimierung nach sich. Grenzen sind

38 Krause, Johannes (2009): „Das Sterben an den EU-Außengrenzen. Die Normalität in der Anormalität." Dossier Border Politics - Migration in the Mediterranen, Berlin, S. 26-31.

39 Gerade unter den derzeitigen Bedingungen ist die Süd-Nord-Migration hingegen nur für den Mittelstand und reichere Bevölkerungsschichten überhaupt eine Option (vgl. Krause, 2009, a.a.O.)

40 Vollmer, Ruth (2014). „Migration im Fokus der Medien: die Zeit des ,Arabischen Frühlings'“. In Erik Bettermann und Ronald Grätz (Hrsg.). Zwischen den Fronten. Grenzen neutraler Berichterstattung. Göttingen: Steidl Verlag (im Erscheinen).

41 De Haas, 2013, a.a.O.

42 Thränhardt, Dietrich (2012): „Neue Grenzen in der Globalisierung. Warum Staaten wieder Mauern bauen." INDES 4 / 2012, S. 14-22. 
nicht nur als Produkte der sie prägenden Gesellschaften zu sehen, sie sind gleichzeitig „Produzenten einer diese Gesellschaft gestaltenden sozialen Ordnung. " ${ }^{43}$ Während offene Grenzen die eigene Sicherheit mit der von Nachbarstaaten verknüpfen und zu engerer Zusammenarbeit führen, führen geschlossene Grenzen zur Konstruktion von Räumen des Chaos und der Gefahr außerhalb. ${ }^{44}$

Ebenfalls ein internationaler Trend, der auch die EU betrifft, ist die Verhandlung (irregulärer) Migration als ein technisch lösbares Problem. Damit wird die politische Dimension in all ihrer Konflikthaftigkeit ausgeklammert, was auf den ersten Blick Vorteile versprechen mag. Wenn jedoch die technischen Lösungen nicht zu dem gewünschten Ergebnis führen, ergibt sich daraus häufig der Ruf nach „komplexere[n], teurere[n], technisch perfektere[n] Lösungen “45, statt nach Alternativen. Laut Thränhardt kommt hier die rituelle Natur von Politik (nach Edelmann) zum Tragen, die über symbolische Akte eine (emotionale) Beeinflussung der Öffentlichkeit zu erzielen sucht und weniger auf die politische Verhandlung und Bearbeitung des eigentlichen Problems bedacht ist. Einiges deutet auch darauf hin, dass die Hersteller von Sicherheitstechnologien eine nicht unerhebliche Rolle für die Beliebtheit hochtechnisierter Lösungen, die nicht zuletzt über europäische Sicherheitsforschungsprogramme wie FP7 gefördert wurden und Einfluss auf die Risikowahrnehmung und Auswahl der Handlungsmöglichkeiten politischer Akteure nehmen können, spielen. ${ }^{46}$

\section{Alternativen}

Grenzen sind keine naturgegebenen Tatsachen, sie werden durch (Kontroll-)Praktiken kreiert und verändert und durch Staat und Gesellschaft mit sich verändernden symbolischen Bedeutungen versehen. ${ }^{47}$ Die EU selbst ist ein Beispiel für diese Wandelbarkeit, da durch den Prozess der europäischen Einigung an ehemals umkämpften und befestigten Grenzen heute nicht einmal mehr Kontrollen stattfinden, während sich der geografische Verlauf ihrer Außengrenzen mit jeder Aufnahmerunde weiter verschiebt. Ob die Steuerung und Kontrolle von Migration entlang von Grenzen aus ethischer Sicht überhaupt $\mathrm{zu}$ rechtfertigen ist, ist im Grunde eine offene Frage. ${ }^{48}$ Dahinter steht der inhärente Widerspruch zwischen universeller Gültigkeit von Menschenrechten und demokratischer Selbstbestimmung. Letztere bestimmt auch über die Zugehörigkeit zu politischen Gemeinschaften, was unweigerlich Ungleichheit schafft. ${ }^{49}$ Fragen von Migration und Grenzkontrollen werden insofern immer in konfliktiven Prozessen ausgehandelt, fak-

43 Eigmüller, Monika (2007): Grenzsicherungspolitik. Funktion und Wirkung der europäischen Außengrenze. Wiesbaden: VS Verlag für Sozialwissenschaften.

44 Marx, Manuel (2013): „Räume, Grenzen. Narrative. Die mediale Verfestigung der europäischen Außengrenze." entgrenzt Nr. 6 2013/14, S. 5-13.

45 Thränhardt, 2013, a.a.O.

46 Hayes, 2009, a.a.O.

47 Andersen, Dorte Jagetic und Sandberg, Marie (2012): „Introduction.“ In Martin Klatt, Marie Sandberg und Dorte Jagetic Andersen (Hrsg.): The Border Multiple. The Practicing of Borders between Public Policy and Everyday Life in a Re-scaling Europe. Surry: Ashgate, S. 1-22.

48 Bader, Veit (2012): "Moral, Ethical, and Realist Dilemmas of transnational Governance of Migration." American Behavioral Scientist, Vol. 56, No. 9, S. 1165-82.

49 Benhabib, Seyla (2008): Kosmopolitismus und Demokratie. Eine Debatte. Frankfurt: Campus Verlag. tisch ist jedoch auch heute noch die Durchsetzbarkeit von Grund- und Menschenrechten stark dem Einfluss von Staatsangehörigkeit und Territorialität unterworfen. ${ }^{50}$ Durch Flucht verlieren Menschen auch heute noch „das Recht Rechte zu haben" bzw. die Möglichkeit, ihre Rechte geltend zu machen, 51 wie „Lampedusa“ gezeigt hat. Gerade weil innerhalb der EU überwiegend eine andere Rechtsauffassung praktiziert wird, derzufolge verbriefte Menschenrechte nicht nur auf dem Papier existieren, sondern durch effektive Durchsetzungsmechanismen mit aufschiebender Wirkung abgesichert sein müssen, besteht die Tendenz der Auslagerung von Migrationskontrollen, die dieses Problem verschärft.

Um dem entgegenzusteuern, wären eine institutionelle Neuaufstellung sowie eine kognitive Neuorientierung in Bezug auf Migration erforderlich. Auf institutioneller Ebene müssten die Verhandlungs- und Entscheidungsprozesse, die bisher stark durch den Rat der Innenminister bestimmt waren, für neue stakeholder geöffnet werden. ${ }^{52}$ Erfolgversprechender wäre es, die Verantwortlichkeit in Arbeits- und Sozialministerien anzusiedeln, wo sie teilweise früher bereits lag. ${ }^{53}$ Auf EU-Ebene wäre etwa eine stärkere Befassung der Generaldirektionen Justiz und Entwicklung mit der Thematik wünschenswert. Im Sinne der Wiederherstellung eines Primats der Politik ist auch der (Teil-) Privatisierung von Kontrollpraktiken und Entscheidungsprozessen entgegenzusteuern.

Als erster Schritt muss tatsächlich, wie in dem Task-Force-Bericht angemahnt, die Kriminalisierung der Seenotrettung durch Italien zurückgenommen und internationales Recht, das diese vorschreibt, wieder eingehalten werden. ${ }^{54}$ Die sogenannten Bossi-Fini-Gesetze, die für diesen Missstand verantwortlich zeichnen, stehen in Italien nicht erst seit „Lampedusa“ in der Kritik. Sie sind jedoch auch vor dem Hintergrund der DublinVerordnung zu sehen, die $u$. a. regelt, dass Asylverfahren im Staat der Ersteinreise stattzufinden haben. Dies trifft auf viele der nördlichen Mittelmeeranrainerstaaten naturgemäß besonders häufig zu und führt zu einer ungleichen und kaum begründbaren Verteilung von Asylbewerbern auf die Mitgliedstaaten. Mit der Verabschiedung der Dublin-III-Verordnung ${ }^{55}$ wurde zwar auf zentrale Kritikpunkte an den Dublin-Überstellungen aus menschenrechtlicher Sicht reagiert, die Regelung der $\mathrm{Zu}$ ständigkeit nach Ersteinreise blieb jedoch in Kraft. Wo die Verordnung de facto schon außer Kraft gesetzt ist (seit 2011 sind Rückführungen aus Deutschland nach Griechenland wegen der dortigen schlechten Menschenrechtssituation ausgesetzt), ${ }^{56}$

50 Buckel und Wissel, 2010, a.a.O.

51 Arendt, Hannah (1955): Elemente und Ursprünge totalitärer Herrschaft. Frankfurt am Main: Pieper.

52 Lavenex und Kunz, 2008, a.a.O.

53 Bommes, 2011, a.a.O.

54 Fischer-Lescano, Andreas und Löhr, Tillmann (2007): Border Controls at Sea: Requirements under International Human Rights and Refugee Law. Berlin: ECCHR.

55 EU Regulation No 604/2013 of the European Parliament and of the Council, http://eur-lex.europa.eu/LexUriServ/LexUriServ. do?uri=OJ:L:2013:180:0031:0059:EN:PDF (eingesehen am 6.2.2014).

56 Vgl. den entsprechenden Erlass des Bundesinnenministeriums hier: http:// www.fluechtlingsrat-mv.de/wp-content/uploads/2014/01/131216 BAMFErlass_Verl\%EF\%BF\%BDngerung-Aussetzung.pdf (eingesehen am 6.2.2014). Im Dezember 2011 urteilte der Europäische Gerichtshof, dass Asylbewerber grundsätzlich nicht in einen EU-Mitgliedstaat überstellt werden dürfen, wenn dort ihre Grundrechte nicht gewährleistet werden können (Text des Urteils hier: http://curia.europa.eu/juris/document/document.jsf? text $=\&$ docid $=117187 \&$ pageIndex $=0 \&$ doclang $=E N \& \operatorname{mode}=1$ st $\&$ dir $=$ \&occ $=$ first\&part $=1 \&$ cid $=564482$, eingesehen am 6.2.2014). 
wird der Druck auf die zuständigen Grenzschutzbehörden verstärkt, mit teilweise erschreckenden Folgen. ${ }^{57}$ Ein Ausgleichssystem für die Aufnahme von Flüchtlingen ist eigentlich seit dem Vertrag von Amsterdam vorgesehen, wurde aber bisher nicht realisiert. In Deutschland wird die Verteilung von Asylbewerbern auf die Bundesländer durch den sogenannten Königsteiner Schlüssel geregelt, in den die Faktoren Bevölkerungszahl und Wirtschaftskraft einfließen. Nun wurden für die EU Berechnungen zu einem Mehrfaktorenmodell vorgelegt, das zusätzlich zu Bevölkerungszahl und Bruttoinlandsprodukt mit geringeren Gewichtungen die Faktoren Fläche und Arbeitslosigkeit mit einfließen lässt. ${ }^{58}$ Die Autoren wollen diesen als Vorschlag für ein faireres Verteilungsverfahren oder als Berechnungsgrundlage für Ausgleichszahlungen verstanden wissen. ${ }^{59}$ Ein solcher Verteilungsschlüssel wäre ein wichtiger Schritt hin zum Abbau zentraler Motivationen hinter den entgrenzten Grenzkontrollen.

Neben der ungleichen Verteilung herrschen jedoch derzeit auch in der Durchführung von Asylverfahren massive Unterschiede zwischen den Mitgliedstaaten in Bezug auf das Verfahren an sich, die Unterbringungsbedingungen und die Anerkennungsquoten vor. Hier sollte das Gemeinsame Europäische Asylsystem Abhilfe schaffen und es wird bei Inkrafttreten der entsprechenden Teile 2015 auch eine gewisse Angleichung bewirken. Die Spielräume für die einzelnen Staaten bleiben aber nach wie vor groß. Hier wird deutlich, dass eine wirkliche Lösung in Europa ansetzen muss, die alleinige Verantwortlichkeit der Ersteinreiseländer für Asylverfahren sollte beendet und Gelder aus dem neu aufgelegten Asyl- und Migrationsfonds (2014-2020) für die Unterstützung der schnellen Angleichung der Asylsysteme auf hohem Niveau bereitgestellt werden.

Kurzfristig müsste auch die Asylantragstellung oder alternativ die Vergabe von Visa aus humanitären Gründen (zur Asylantragstellung) in Botschaften und Auslandsvertretungen vor Ort möglich gemacht werden (letzteres ist nach geltendem EU-Recht bereits möglich). Zu vermeiden ist dabei eine Auslagerung des Asylverfahrens, nicht nur um politisch Verfolgte nicht weiter zu gefährden, sondern auch, weil vor Ort der benötigte - auch gerichtliche - Rechtsschutz während des Verfahrens nicht gewährleistet werden kann.

Was hingegen häufig von Seiten der Politik als Lösungsvorschläge vorgebracht wird, nämlich die Vertiefung der wirtschaftlichen Zusammenarbeit mit afrikanischen Staaten, die Forderung nach mehr Entwicklungszusammenarbeit und die Bekämpfung von Fluchtursachen, ist kritischer zu bewerten. Sie entspringen dem oben skizzierten framing von Migration und ihnen liegt somit die Annahme zu Grunde, der beste Umgang mit unkontrollierten Wanderungsbewegungen bestehe in ihrer Verhinderung. Im Fall von Flucht und Vertreibung ist das nicht ganz falsch, sicherlich sollten verstärkt Bemühungen unternommen werden, deren Ursachen zu beseitigen. Allerdings zeigt etwa der Blick nach Syrien, dass es sich hier nicht um eine Alternative zur Aufnahme von Flüchtlingen handeln kann, sondern höchstens um eine zusätzliche Bemühung. Auch im

57 Pro Asyl, 2013, a.a.O.

58 Angenendt, Steffen, Engler, Marcus, Schneider, Jan (2013): Europäische Flüchtlingspolitik. Wege zu einer fairen Lastenteilung. SWP Aktuell Nr. 65. 59 Ebenda. vierten Jahr andauernder Gewalt und Vertreibungen herrscht Ratlosigkeit, wie diese zu beenden seien.

Im Zusammendenken von Migration und Entwicklung liegen durchaus Potenziale für beide Bereiche. ${ }^{60}$ Dahinter verbergen sich jedoch sehr unterschiedliche Vorstellungen. Während auf internationaler Ebene durch Organisationen wie die ILO und die IOM die Ermöglichung und Steuerung von Migration mit dem Ziel einer positiven Entwicklungswirkung vertreten wird, hat sich in der praktischen Auseinandersetzung der EUInstitutionen mit dem sogenannten Migrations-EntwicklungsNexus eher die Förderung von Entwicklung zur Begrenzung von Migration durchgesetzt. ${ }^{61}$ Entwicklungspolitische Maßnahmen mit der so zu erzielenden Verhinderung von Migration zu begründen, trägt nicht nur zu einer Problematisierung von Migration bei, sondern ist auch sachlich falsch. Ein steigendes Bruttoinlandsprodukt in sogenannten Entwicklungsländern führt nach allen bisherigen Erkenntnissen zunächst zu einer Zunahme von Abwanderung, nicht zu deren Rückgang, da mehr Menschen in den Besitz der dafür erforderlichen Ressourcen gelangen. ${ }^{62}$ Sinnvoll wäre eher die Ermöglichung legaler, längerfristiger Arbeitsmigration Geringqualifizierter, nicht nur weil hier ein Bedarf kaum bestreitbar ist - der ökonomische Nutzen ist nachgewiesen. ${ }^{63}$ Die Legalisierung würde auch effektivere Möglichkeiten eröffnen, um gegen Ausbeutung vorzugehen, als die derzeitigen strafrechtlichen Maßnahmen. ${ }^{64}$ Wirtschaftliche Zusammenarbeit ist sinnvoll, wenn beiderseitigen Interessen Rechnung getragen wird. In der Vergangenheit war jedoch häufiger ein Zusammenhang zwischen entsprechenden Interventionen der Zielländer und verstärkter Abwanderung zu beobachten. Der Beitrag von „Empfängerländern“ internationaler Migration zu deren Entstehen ${ }^{65}$, z.B. durch unfaire weltwirtschaftliche Verhältnisse, wird bei solchen Vorschlägen ignoriert.

In langfristiger Sicht wäre es generell ratsam, in Zusammenarbeit mit außereuropäischen Nachbarstaaten gemeinsame Interessen $\mathrm{zu}$ identifizieren und zu verfolgen und in diesem Rahmen Mobilitätsförderung zu betreiben, die den Namen auch tatsächlich verdient. Der Abbau von Migrationshürden würde sicherlich zu einer Zunahme dauerhafter Einwanderung führen. Vor allem aber zeigt die Erfahrung, dass so zirkuläre Migration, also ein Pendeln zwischen Herkunfts- und Zielland und vielfältige, nicht auf Dauer angelegte Mobilitätsformen gefördert werden, ${ }^{66}$ sodass eine allgemeine Lockerung der VisaVergabepraxis sinnvoll ist. Wie Thränhardt schreibt, hat die EU das Potenzial weltweit zu demonstrieren, wie durch zwischenstaatliche Kooperation Probleme gelöst werden können, statt durch Abschottung neue zu schaffen. ${ }^{67}$

60 Schraven, Benjamin, Keijzer, Niels und Knoll, Anna (2013): "Post 2015: Making Migration Work for Sustainable Development." DIE Briefing Paper $21 / 2013$.

61 Lavenex und Kunz, 2008, a.a.O.

62 De Haas, Hein (2007): "Turning the Tide? Why Development Will Not Stop Migration." Development and Change, Vol 38, No. 5.

63 Schraven et al., 2013, a.a.O.

64 De Haas, 2013, a.a.O.

65 Sassen, Saskia (2013). Migration in einer begrenzten Welt. epd-Dokumentation $\mathrm{Nr} 40,2013$, Frankfurt a.M., S. 10-14.

66 Sassen, Saskia (2000): "Regulating Immigration in a Global Age: A New Policy Landscape." The ANNALS of the American Academy of Political and Social Science, 570, S. 65-77.

67 Thränhardt, 2012, a.a.O. 
Die eigentliche Herausforderung stellt sich nicht an den Außengrenzen, sie stellt sich zum einen in den Köpfen und zum andern für die Systeme der sozialen Wohlfahrt, die nationalstaatlich organisiert sind. Die Erfahrung, dass Migration durch den Staat nur bedingt plan- und steuerbar ist, darf nicht dazu verleiten, sie unter hohem organisatorischem Aufwand im „Modus der Gefahrenabwehr" zu erschweren, zumal wenn sich die Gefahrenabwehr dabei gegen die gefährdeten Personen und nicht gegen die Gefahren richtet. ${ }^{68}$ Vielmehr muss Migration im Sinne der tatsächlich stattfindenden Bewegungen gerade angesichts ihrer Unkalkulierbarkeit als eine politische Gestaltungsaufgabe begriffen werden. Dafür gilt es, sich von der gedanklichen Gegenüberstellung „nützlicher“ Einwanderung Hochqualifizierter und Asyl- und Fluchtmigration als vermeintlicher Belastung zu verabschieden. Eine Politik, die dies umsetzt, würde die Potenziale aller Migrantinnen und Migranten in den Blick nehmen und fördern. Ein mögliches Modell dazu, das sich global für die Einführung eines Grundrechts auf Mobilität

68 Bommes, 2011, a.a.O. ausspricht, sieht vor, die bisher überwiegend an die Staatsangehörigkeit geknüpften Rechte in politische, bürgerliche, soziale, familiäre, kulturelle Rechte zu unterteilen. ${ }^{69}$ Neuankömmlingen könnten zunächst unmittelbar bestimmte fundamentale bürgerliche und soziale Rechte übertragen werden, weitergehende Ansprüche auf soziale Leistungen, Teilnahme an Wahlen, Zusammenleben mit der Familie, etc., die derzeit an Vorhandensein und Art des Aufenthaltstitels gebunden sind, würden davon unabhängig nach einer festzulegenden Zeit übertragen. Die Binarität zwischen Inklusion und Exklusion würde so aufgehoben und die rechtliche Schlechterstellung irregulärer Einwanderer wäre abgemildert. Dies würde erlauben, die erwiesenen Vorteile internationaler Migration, ${ }^{70}$ auch in wirtschaftlicher und wohlfahrtsstaatlicher Hinsicht, und die humanitäre Schutzverantwortung europäischer Staaten in Einklang zu verwirklichen.

69 Pécoud, Antoine und de Guchteneire, Paul (2006): “International Migration, Border Controls and Human Rights: Assessing the Relevance of a Right to Mobility." Journal of Borderland Studies, Vol. 21, No 1, S. 69-86.

70 UNDP (2009): "Overcoming Barriers: Human Mobility and Development." Human Development Report 2009, New York: Palgrave Macmillan.

\section{Bilanz der europäischen Integration}

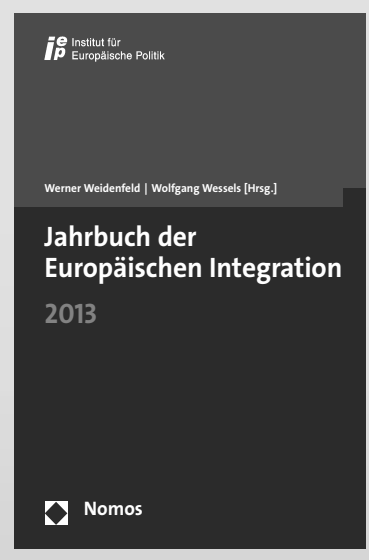

Das Jahrbuch der Europäischen Integration des Instituts für Europäische Politik (Berlin) dokumentiert und bilanziert seit 1980 zeitnah und detailliert den europäischen Integrationsprozess. Entstanden ist in 33 Jahren eine einzigartige Dokumentation der europäischen Zeitgeschichte. Das „Jahrbuch der Europäischen Integration 2013“ führt diese Tradition fort.

\section{Jahrbuch der Europäischen Integration 2013}

Herausgegeben von Werner Weidenfeld und Wolfgang Wessels

2013, 538 S., brosch., 64,- $€$

ISBN 978-3-8487-0518-4

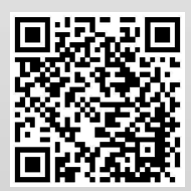

Kostenlose Leseprobe
In über 90 Beiträgen zeichnen die Autorinnen und Autoren in ihren jeweiligen Forschungsschwerpunkten die europapolitischen Ereignisse des Berichtszeitraums 2012/2013 nach und informieren über die Arbeit der europäischen Institutionen, die Entwicklung der einzelnen Politikbereiche der EU, Europas Rolle in der Welt und die Europapolitik in den Mitgliedstaaten und Kandidatenländern. 